LA-UR 05-4013

astro-ph/0506395

DRAFT VERSION NOVEMBER 12, 2018

Preprint typeset using LTEX style emulateapj v. 11/26/04

\title{
PRECISION DETERMINATION OF THE MASS FUNCTION OF DARK MATTER HALOS
}

\author{
Michael S. WArren ${ }^{1}$, Kevork AbaZajian ${ }^{1}$, Daniel E. Holz ${ }^{1}$ and Luís Teodoro ${ }^{1,2}$ \\ Draft version November 12, 2018
}

\begin{abstract}
The predicted mass function of dark matter halos is essential in connecting observed galaxy cluster counts and models of galaxy clustering to the properties of the primordial density field. We determine the mass function in the concordance $\Lambda \mathrm{CDM}$ cosmology, as well as its uncertainty, using sixteen $1024^{3}$-particle nestedvolume dark-matter simulations, spanning a mass range of over five orders of magnitude. Using the nested volumes and single-halo tests, we find and correct for a systematic error in the friends-of-friends halo-finding algorithm. We find a fitting form and full error covariance for the mass function that successfully describes the simulations' mass function and is well-behaved outside the simulations' resolutions. Estimated forecasts of uncertainty in cosmological parameters from future cluster count surveys have negligible contribution from remaining statistical uncertainties in the central cosmology multiplicity function. There exists a potentially non-negligible cosmological dependence (non-universality) of the halo multiplicity function.

Subject headings: cosmology: theory — galaxies: clusters: halos — galaxies: halos
\end{abstract}

\section{INTRODUCTION}

Collapsed, virialized dark matter halos arise from density peaks in the initially Gaussian primordial fluctuation field (Press \& Schechter 1974; Bardeen et al. 1986). The abundance of the most massive of these halos is exponentially sensitive to the amplitude of the initial fluctuation field as well as the mean matter density, making observed counts of their abundance extremely sensitive to these properties of the density field, as well as the dark-energy dependent growth rate of the density field (e.g., Haiman et al. 2001). Condensation of gas and formation of stars within these halos leads to the formation of galaxies (White \& Rees 1978). In addition, nonlinear clustering halo-models of dark matter and galaxies require, as a basic component, the mass function (see Cooray \& Sheth 2002 for a recent review).

The analytic theory of virialized object formation through collapse of overdense regions as envisioned by Press \& Schechter (1974) (hereafter PS) employs the fact that a uniform overdensity in the Universe will evolve as a separate, closed universe, initially expanding with the background, but then slowing and turning around to collapse and virialize. Since the abundance of overdensity peaks only depends on the fluctuation scale $\sigma$, the abundance of halos can be expected to be universal in these units. Limitations of approximations in the PS model, e.g., sphericity of collapse and spatial overlap, led to a modification of the original form with parameters fit to simulations (Sheth \& Tormen 1999, hereafter ST). Using the same simulations, Jenkins et al. (2001) (hereafter J01) abandoned the form of the PS motivated mass function to better fit the simulations' mass range, but their functional form cannot be extrapolated beyond the range of the fit. J01 found the mass function in $\sigma$ to be approximately universal for several cosmologies at the level of $\sim 15 \%$.

Here, we present a quantification of the dark matter halo mass function and its uncertainties with a suite of sixteen nested-volume $1024^{3}$ particle dark matter simulations of the

\footnotetext{
${ }^{1}$ Theoretical Division, Los Alamos National Laboratory, Los Alamos, NM 87545

${ }^{2}$ Department of Physics, Kelvin Building, University of Glasgow, G12 8QQ, Glasgow, Scotland, UK
}

concordance $\Lambda \mathrm{CDM}$ cosmology. We quantify the uncertainty and full covariance of the mass function parameters. Our halo-finding methodology is given in $\$ 2$, mass function determination and error analysis is presented in $\$ 3$ along with implications for future cluster surveys' sensitivities; we present our conclusions in $\$ 4$

\section{NUMERICAL SIMULATIONS AND HALO MASS DETERMINATION}

We calculate the mass function of dark matter halos arising in a concordance $\Lambda$ CDM model by performing numerical simulations of structure growth and halo formation. We use the Hashed-Oct-Tree (HOT) algorithm, initially described in Warren \& Salmon (1993) and recently compared in detail with other well-known cosmology codes in Heitmann et al. (2004). We use a per-interaction error bound based on the analysis in Salmon \& Warren (1994). The fractional error per interaction is set to be no worse than $10^{-5}$ at a redshift of 25 , increasing to $10^{-3}$ at redshifts of 5 and lower. The number of timesteps and Plummer smoothing lengths ranged from 1480 steps and $2.1 h^{-1} \mathrm{kpc}$ (physical) for the highest resolution simulation, to 720 steps and $98 h^{-1} \mathrm{kpc}$ (comoving) for the largest volume. Each simulation required about $2 \times 10^{17}$ floating point operations, which can be computed in roughly 60 hours on a 1024 processor parallel computer using HOT. Overall, the results presented here required over four exaflops ( $4 \times 10^{18}$ floating point operations).

We model a universe with flat geometry and parameters

$$
\mathbf{p}=\left(\Omega_{M}, \Omega_{b}, n, h, \sigma_{8}\right)=(0.3,0.04,1,0.7,0.9) \text {. }
$$

Initial conditions are derived from the transfer functions as calculated by CMBFAST (Seliak \& Zaldarriaga 1996).

In order to simultaneously reduce Poisson error, verify consistency, and resolve the widest mass-scale range available by our techniques, we employ nested volumes with independent realizations of the chosen cosmology. We simulated sixteen boxes of sizes $96,135,192,272,384,543,768,1086,1536$, 2172,2583 and $3072 h^{-1} \mathrm{Mpc}$, with three realizations of 384 $h^{-1} \mathrm{Mpc}$ size, and two each of 272 and $3072 h^{-1} \mathrm{Mpc}$. After the aggressive requirement of a minimum of 400 particles per halo, we measure the mass function over five orders of magnitude in mass scale; see Fig. (1). The box size minimum is 


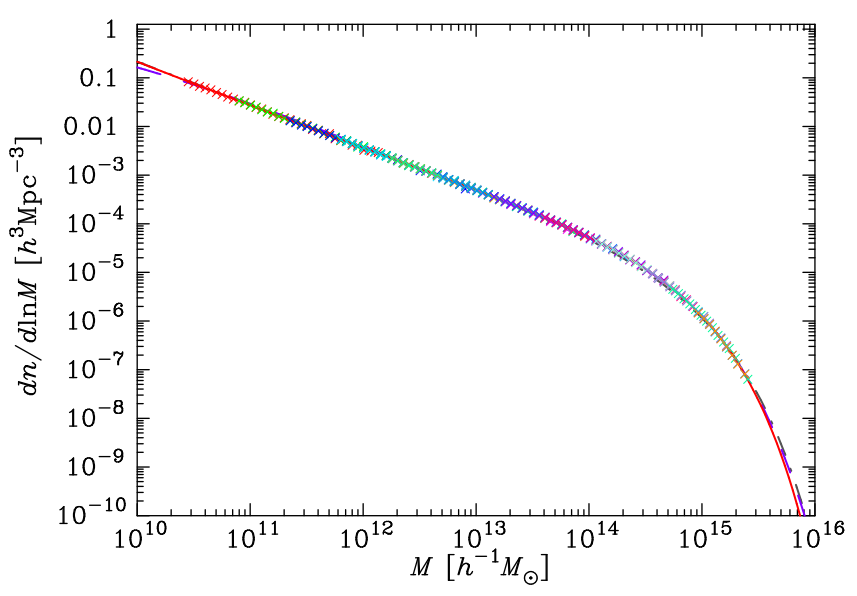

FIG. 1. - Shown are the central values of the binned mass functions from sixteen $1024^{3}$ simulations of the $\Lambda$ CDM universe as crosses, with simulations in different colors. The best-fit form for the mass function we calculate is in solid (red), the Jenkins fit dashed (purple), and the Sheth-Tormen fit in dotdashed (dark grey). Goodness-of-fit is poorly judged on this extreme log scale; it is more clearly resolved in the linear residuals of Fig. 2

set by requiring the largest scale growth modes in the volume to remain linear, while the the maximum is set by the halo particle number requirement (i.e., very few massive halos in boxes of size $>3 h^{-1} \mathrm{Gpc}$ have more than 400 particles). Our nested-volume approach allows greater mass and length-scale resolution than pure particle number and force resolution increase within a single simulation (e.g., Springel et al. 2005).

The friends-of-friends (FOF) method (Frenk et al. 1988) identifies a set of particles which are spatially associated and contained within a given isodensity surface defined by a linking parameter, $b$. The linking length is defined as $h_{\text {link }}=$ $b n^{-1 / 3}$, where $n$ is the number density of particles.

In practice, the number of particles per halo is not sufficient to suppress statistical noise in the density field represented by the finite number of particles. This leads to a significant systematic error in the estimated mass of a given halo, which depends on the number of particles which represent the halo. As an illustration of one aspect of this problem, consider an isothermal density distribution of total mass $M$ represented by $N$ particles within a sphere of radius R. Each particle has mass $M_{p}=M / N$. Choosing an FOF link length $h_{\text {link }}$ defines an isodensity surface with value

$$
\rho\left(h_{\text {link }}\right)=\frac{\alpha M_{p}}{(4 \pi / 3) h_{\text {link }}^{3}},
$$

where $\alpha$ is "a constant of order 2" (Frenk et al. 1988).

Using the FOF method on a large number of sample isothermal halos with parameters $M=R=1$ and $h_{\text {link }}=(N / 1.25)^{-1 / 3}$ while varying $N$ leads to the results that are presented in Table 1 It is clear from these results that there is a strong $N$ dependence in the determined mass of the same underlying density distribution. Another interesting result is that the mass values are converging to a value which is significantly different from that implied from Eq. (2) with $\alpha=2.0$. For large $N$, the correct value for $\alpha$ is close to 3.1, which implies that the overdensity of halos being found with the commonly favored link parameter of $b=0.2$ is about $280 \rho_{b}$, rather than the often quoted value of $180 \rho_{b}$ (Cole \& Lacev 1994; Jenkins et al. 2001; White 2001, 2002).

For a well-behaved halo finding method, one should be able to sub-sample a simulation and derive the same mass function in the regime where the statistics are robust. In the case of FOF, this means that one should be able to randomly pick one

\begin{tabular}{rccrcc}
\multicolumn{1}{c}{$N$} & $M_{F O F}$ & $\sigma$ & \multicolumn{1}{c}{$N$} & $M_{F O F}$ & $\sigma$ \\
\hline 640000 & 0.3879 & 0.0014 & 500 & 0.4704 & 0.0371 \\
80000 & 0.3999 & 0.0037 & 200 & 0.4964 & 0.0555 \\
10000 & 0.4187 & 0.0092 & 100 & 0.5222 & 0.0765 \\
1250 & 0.4513 & 0.0244 & 50 & 0.5586 & 0.1018 \\
\hline
\end{tabular}

TABLE 1

THE MEAN AND VARIANCE OF FOF MASS VS $N$ FOR AN IDEAL HALO

of every $n$ particles in the simulation, run the FOF method with a link length $\sqrt[3]{n}$ times as large, and find the same distribution of halos.

However, due to the $N$-dependent statistical effects described above, one obtains substantially different massfunctions from a sub-sampled distribution. While it is possible to use the binomial distribution to determine an expression for the $N$-dependent corrections to the FOF method in the case of an ideal isothermal density distribution, halos found in simulations are complex objects which are not well-described by such a smooth density distribution. For this reason, we have chosen to implement a correction which is calibrated via the comparison of a simulation to the sub-sampled version of itself. Fortunately, the correction derived from such a procedure appears to not depend strongly on the parameters of the simulation. We find a correction of the form

$$
N_{\text {corrected }}=N\left(1-N^{-0.6}\right),
$$

for a halo with $N$ particles to do a reasonable job of correcting the systematic error caused by the FOF method.

With this correction and the understanding of the behavior of FOF for highly-resolved halos discussed above, for a link parameter $b=0.2$ (used for all further analysis in this paper) we find that the mean density of halos with respect to the background in our largest volume simulation is $260 \pm 50$, while in the smallest volume this value is $340 \pm 60$. For this reason, further analysis based on the results described below should not assume that the overdensity of FOF halos is constant with respect to scale. The process of defining a halo remains the largest uncertainty in defining the mass function. New algorithms for halo finding based on an overdensity criterion rather than the use of FOF will be necessary for straightforward comparisons with observational data.

\section{THE MASS FUNCTION}

The relation between the multiplicity function $f(\sigma)$ and mass function $n$ is

$$
f(\sigma)=\frac{M}{\bar{\rho}} \frac{d n}{d \ln \sigma^{-1}},
$$

where $\bar{\rho}$ is the mean matter density of the universe. The general shape of the mass function is well described by the PS and ST forms for the multiplicity function. We therefore use a similar form for the mass function as that of ST, with a disentanglement of the power-law small-mass regime from the exponential cut-off, and removal of the arbitrary collapse scale $\delta_{c}$, which was nevertheless modified by the ellipsoidal collapse scale in the ST model. The minimal-parameter multiplicity function required to fit the mass-function shape measured in the simulations is

$$
f(\sigma)=A\left(\sigma^{-a}+b\right) e^{-c / \sigma^{2}},
$$

with parameters $\mathbf{q}=(A, a, b, c)$. This form is related to the multiplicity function resulting from the barrier shape ansatz of Sheth \& Tormen (2002), but without an associated change of the exponential. 


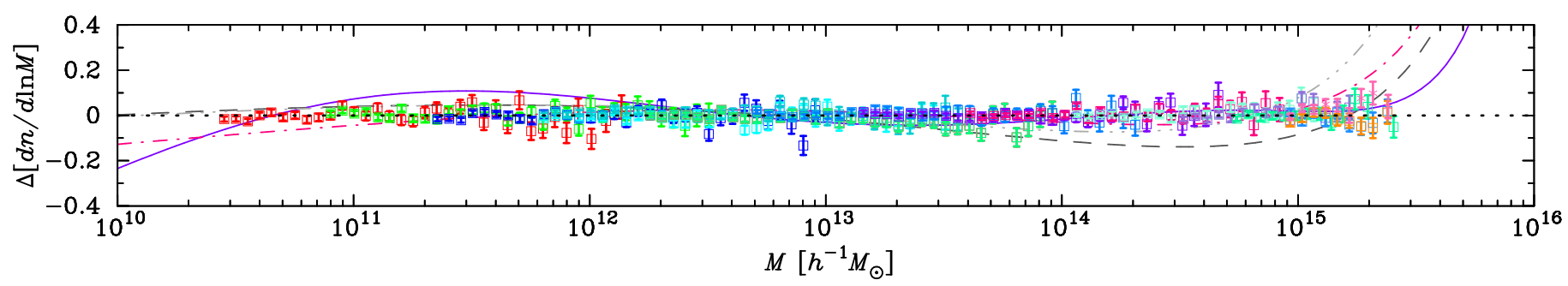

FIG. 2.- Shown are the residuals from the binned simulation data to the fit presented in this work as square data points of different colors per simulation. The Jenkins fit is the solid (purple) line, ST original fit the dashed (dark gray) line, the ST fit with parameters $A, a, p$ free with dot-dashed line (red), and the ST fit with $a, p$ free and amplitude $A$ set to require all dark matter in halos as a triple-dot-dashed line (light gray). The binned mass function from the Virgo Hubble Volume simulation are the asterisk points with errors (pink).

Using, Eq. (5), we calculate the extended maximum likelihood, $\lambda(\mathbf{q})$ for the Poisson data of the mass function found in the combined set of simulations (Eidelman et al. 2004),

$$
\ln \lambda(\mathbf{q})=-\sum_{j=1}^{N_{\mathrm{s}}} \sum_{i=1}^{N_{j}}\left[\mu_{i j}(\mathbf{q})-n_{i j}+n_{i j} \ln \frac{n_{i j}}{\mu_{i j}(\mathbf{q})}\right],
$$

of $N_{\mathrm{s}}$ simulations containing $N_{j}$ bins, where $n_{i j}$ is the number of halos in mass bin $i$ of simulation $j$. In bins where $n_{i j}=0$ the last term is zero. The predicted number of halos in bin $i$ of simulation $j$ between masses $m_{i j 1}$ and $m_{i j 2}$ is

$$
\mu_{i j}(\mathbf{q})=V_{j} \int_{m_{i j 1}}^{m_{i j 2}} \frac{d n(\mathbf{q})}{d M} d M
$$

where $V_{j}$ is the volume of the simulation. The extended maximum likelihood is appropriate for Poisson data sets such as the mass function, and allows for an estimation of the goodness-of-fit for a given model, with the number of degrees of freedom for the multinomial nature of the distribution given by $N-k-1$, where $N$ is the total number of bins and $k$ is the number of fitted parameters.

The mass function from all simulations is binned with minimum logarithmic width of 0.05 , with the last bin expanded to include a minimum of 400 halos. Higher mass halos have negligible statistical weight and are discarded. The low-mass end requires 400 particles per halo, and the FOF determined masses are corrected as described in $\$ 2$

Sample variance can be a consideration when attempting to use the most massive halos available in a small volume (e.g., see $\mathrm{Hu} \&$ Kravtsov 2003). The sample variance of halos of mass $M$ in a cubic volume of side length $L$ such as our simulations is $b^{2}(M) \sigma^{2}(L)$, where $b(M)$ is the halo bias and $\sigma(L)$ is the fluctuation amplitude in the cubic volume. For our simulation volume samples, the maximum sample variance is $0.2 \%$ of the Poisson error, which occurs for the most massive halo bin in our smallest volume simulation. Sample variance is therefore negligible here.

We perform an analysis of the likelihood, Eq. (6), for the multiplicity function, Eq. (5), to obtain the simulations' results for the parameters $\mathbf{q}$ free. The best-fit of the multiplicity function is found with parameters

$$
\begin{aligned}
& A=0.7234 \pm 0.0043 \text { (stat.) } \pm 0.003 \text { (sys.), } \\
& a=1.625 \pm 0.019 \text { (stat.) } \pm 0.009 \text { (sys.), } \\
& b=0.2538 \pm 0.0031 \text { (stat.) } \pm 0.002 \text { (sys.), } \\
& c=1.1982 \pm 0.0055 \text { (stat.) } \pm 0.002 \text { (sys.), }
\end{aligned}
$$

with corresponding $1 \sigma$ statistical errors, and estimated systematic uncertainty. The statistical error covariance is

$$
\mathbf{C}=\left(\begin{array}{llll}
1.82 & 5.80 & 0.0730 & 2.04 \\
\ldots & 36.7 & 4.35 & 9.85 \\
\ldots & \ldots & 0.958 & 0.854 \\
\ldots \ldots & \ldots & \ldots \ldots & 2.97
\end{array}\right) \cdot 10^{-5}
$$

Error analysis was performed with the MINUIT package of CERNLIB. The $\chi^{2}$ per degree-of-freedom (DOF) for the best fit is $\chi^{2} / \mathrm{DOF}=524.5 / 429$, indicating a larger scatter than expected from the Poisson statistics.

The uncertainty and resulting scatter in the sampling of a given overdensity by the FOF halo finder likely contributes to the higher than expected $\chi^{2} / \mathrm{DOF}$. To assess this source of systematic uncertainty, we vary the FOF correction Eq. (3) within ranges fit by single simulations, as well as alternate binnings of the mass function, which sample this distribution differently. The estimated systematic uncertainties are subdominant to statistical, though not negligible.

The residuals of the binned mass functions from all simulations to the expected number of halos in the given bin [Eq. (7)] of mass-function best fit are shown in Fig. 2] In this figure, we also plot the binned mass function from the Virgo Hubble Volume simulation (Jenkins et al. 2001), which we derive from their particle data identically to our simulations (although the initial power spectrum for those data are not precisely the same as our simulations).

For comparison, we also fit the ST form for the multiplicity function. With amplitude free of the constraint that all dark matter be within halos, the ST parameters are $A=$ $0.3405 \pm 0.0004, a=0.7183 \pm 0.001$ and $p=0.157 \pm 0.003$, with $\chi^{2} / \mathrm{DOF}=1987 / 430$. From the derived $\chi^{2} / \mathrm{DOF}$ and Fig. 2] modifications of ST form and the J01 form clearly provide a poor fit to the $\Lambda$ CDM mass function. However, the ST parameters are similar to that fit to the bias from simulations of Seliak \& Warren (2004) using the ST form as given in Mandelbaum et al. (2004). Therefore, the peakbackground split ansatz of the relation between halo biasing and the mass function may be consistent (Mo \& White 1996; Sheth \& Tormen 1999; Sheth et al. 2001). Although more can be done to verify this statistical consistency, it is beyond the scope of this Letter.

To test the claim of the multiplicity function's universality in consistently describing varying cosmologies, we find the residuals for the mass function derived from the $\Lambda \mathrm{CDM}$ simulations' multiplicity function versus simulations done for varying cosmological parameters within $\Lambda \mathrm{CDM}$. Four separate $1024^{3}$ simulations were run with parameters as those in Eq. (1) except, a $384 h^{-1} \mathrm{Mpc}$ box with $\sigma_{8}=0.8$; a $384 h^{-1} \mathrm{Mpc}$ box with $\Omega_{m}=0.2$; a $329 h^{-1} \mathrm{Mpc}$ box with $h=0.6$ and $\sigma_{8}=0.8$; and a $1536 h^{-1} \mathrm{Mpc}$ box with $\Omega_{m}=0.2$ and $\sigma_{8}=1.2$. Residuals are shown in Fig. 3. The models are consistent within the $5 \%$ level, except for the $\Omega_{m}=0.2$ and $\sigma_{8}=1.2$ model, which shows departures at $20 \%$. The departure may be more than a Poisson fluctuation, revealing a statistically significant dependence of the multiplicity function on cosmology. Quantifying this potential dependence is warranted, but beyond the scope of this work. 


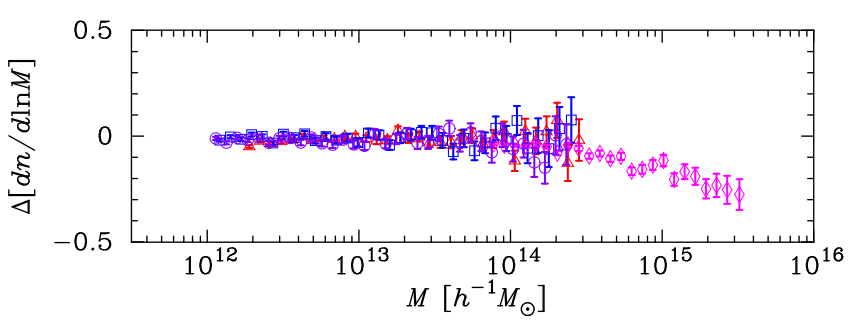

FIG. 3.- Residuals are shown for simulations with varied cosmological parameters with that cosmologies' mass function using the multiplicity function predicted from the central cosmology. Cosmological parameter are held constant, except triangles are for $\sigma_{8}=0.8$; squares are for $\Omega_{m}=0.2$; circles for $h=0.6$ and $\sigma_{8}=0.8$; diamonds are for $\Omega_{m}=0.2$ and $\sigma_{8}=1.2$.

A potential implication of this work is the calculation of the sensitivities of current and future cluster surveys to properties of the primordial perturbations and the dark-energydependent growth of these perturbations. Some of the original work on quantifying parameter sensitivities of future cluster surveys considered the bias associated with mass function uncertainties (Holder et al. 2001; Haiman et al. 2001), as well as in more recent work (Battye \& Weller 2003). However, the uncertainties in the predicted dark matter halo mass function are often entirely ignored (Wang et al. 2004, 2005).

To test the potential implications of dark matter halo mass function uncertainties on forecasts for parameter sensitivity, we use the Fisher matrix approach of Holder et al. (2001) for sensitivities of the South Pole Telescope (SPT) SunyaevZeldovich Effect cluster survey, with redshifts determined by the Dark Energy Survey (DES), and a minimum mass cutoff of $M_{\text {min }}=3 \times 10^{14} h^{-1} M_{\odot}$. Sky coverage is $4000 \mathrm{deg}^{2}$ and cluster counts are binned in redshifts $\Delta z=0.05$ from $z=0$ to $z=2$. We have also calculated and included the Fisher information from forecasts for the Planck mission parameter sensitivities from the cosmic microwave background (CMB) $T T, T E$ and $E E$ correlations (Eisenstein et al. 1998). In this test, we have not included scatter in the masstemperature relation (Levine et al. 2002), nor uncertainty in this distribution (Lima \& Hu 2005), but have also not included mass-observable self-calibration (Majumdar \& Mohr 2003), which can be compensating effects. We find that the inferred uncertainties forecast for parameters best constrained by an SPT+DES cluster survey beyond the CMB constraints, namely the equation of state, $\Omega_{m}$ and neutrino mass, have forecast errors increased by, at most, approximately $1 \%$ in the error. Therefore, the mass function presented here has its uncertainties sufficiently well determined so that they are negligible compared to observational uncertainties.

\section{CONCLUSIONS}

We have measured the shape and quantified the uncertainty in the predicted theoretical mass function of dark matter halos in the $\Lambda$ CDM cosmology with sixteen separate $1024^{3}$ dark matter structure formation simulations. We found and corrected a systematic error in halo mass determination by the friends-of-friends halo finder. The canonical Sheth \& Tormen (1999) and Jenkins et al. (2001) forms of the mass function are inconsistent with the $\Lambda$ CDM mass function at $\sim 10 \%$ level at intermediate masses, and $>30 \%$ at the highest masses.

Combining the work presented here with uncertainties in halo biasing will be necessary for improving work on quantified applications of the halo model (van den Bosch et al. 2003; Abazaiian et al. 2005). The peak-background split model for the relation between halo bias and the mass function appears consistent, although this should be studied in further detail.

Analyses of current and future cluster surveys' sensitivities to the primordial perturbation spectrum and dark energy are not greatly dependent on inherent statistical uncertainties in the predicted dark matter halo mass function. The use of high-redshift cluster count surveys will require quantification of the predicted evolution of the mass function, as well as any cosmological dependence of the halo mass multiplicity function. Simulation efforts may be tailored for a specific survey's requirements in this regard. In concert with observations, this will help elucidate the nature of dark energy, neutrino mass, cosmological structure and galaxy formation.

We would like to thank Josh Frieman, Salman Habib, Katrin Heitmann, Alexia Schulz, Uroš Seljak, Ravi Sheth, Jochen Weller and Simon White for useful conversations. We thank the Virgo Supercomputing Consortium for making available to us their Hubble Volume data. This work was performed under the auspices of the U.S. Dept. of Energy, and supported by its contract \#W-7405-ENG-36 to Los Alamos National Laboratory. The computational resources were provided by the LANL open supercomputing initiative and the Space Simulator Beowulf cluster.

\section{REFERENCES}

Abazajian, K., et al. 2005, ApJ, 625, 613

Bardeen, J. M., Bond, J. R., Kaiser, N., \& Szalay, A. S. 1986, ApJ, 304, 15

Battye, R. A., \& Weller, J. 2003, Phys. Rev., D68, 083506

Cole, S., \& Lacey, C. G. 1994, MNRAS, 271, 676

Cooray, A., \& Sheth, R. 2002, Phys. Rept., 372, 1

Eidelman, S., et al. 2004, Phys. Lett. B, 592, 1

Eisenstein, D. J., Hu, W., \& Tegmark, M. 1998, Astrophys. J., 518, 2

Frenk, C. S., White, S. D. M., Davis, M., \& Efstathiou, G. 1988, ApJ, 327, 507

Haiman, Z., Mohr, J. J., \& Holder, G. P. 2001, ApJ, 553, 545

Heitmann, K., Ricker, P., Warren, M. S., \& Habib, S. 2004, ApJS, in press, astro-ph/0411795

Holder, G., Haiman, Z., \& Mohr, J. J. 2001, ApJ, 560, L111

Hu, W., \& Kravtsov, A. V. 2003, ApJ, 584, 702

Jenkins, A., et al. 2001, MNRAS, 321, 372

Levine, E. S., Schulz, A. E., \& White, M. J. 2002, Astrophys. J., 577, 569

Lima, M., \& Hu, W. 2005, Phys. Rev. D., submitted, astro-ph/0503363

Majumdar, S., \& Mohr, J. J. 2003, Astrophys. J., 585, 603

Mandelbaum, R., Tasitsiomi, A., Seljak, U., Kravtsov, A. V., \& Wechsler, R. H. 2004, MNRAS, submitted, astro-ph/0410711
Mo, H. J., \& White, S. D. M. 1996, MNRAS, 282, 347

Press, W. H., \& Schechter, P. 1974, ApJ, 187, 425

Salmon, J. K., \& Warren, M. S. 1994, J. Comp. Phys., 111, 136

Seljak, U., \& Warren, M. S. 2004, MNRAS, 516

Seljak, U., \& Zaldarriaga, M. 1996, ApJ, 469, 437

Sheth, R. K., Mo, H. J., \& Tormen, G. 2001, MNRAS, 323, 1

Sheth, R. K., \& Tormen, G. 1999, MNRAS, 308, 119

-. 2002, MNRAS, 329, 61

Springel, V., et al. 2005, Nature, 435, 629

van den Bosch, F. C., Mo, H. J., \& Yang, X. 2003, MNRAS, 345, 923

Wang, S., Haiman, Z., Hu, W., Khoury, J., \& May, M. 2005, Phys. Rev. Lett., in press, astro-ph/0505390

Wang, S., Khoury, J., Haiman, Z., \& May, M. 2004, Phys. Rev., D70, 123008

Warren, M. S., \& Salmon, J. K. 1993, in Supercomputing 1993 (Los

Alamitos: IEEE Comp. Sci.), 12

White, M. 2001, A\&A, 367, 27

White, M. 2002, ApJS, 143, 241

White, S. D. M., \& Rees, M. J. 1978, MNRAS, 183, 341 